\title{
Neuronal Mechanisms of Cortical Alpha Oscillations in Awake-Behaving Macaques
}

\author{
Anil Bollimunta, ${ }^{1}$ Yonghong Chen, ${ }^{1}$ Charles E. Schroeder ${ }^{2,3}$ and Mingzhou Ding ${ }^{1}$ \\ ${ }^{1}$ J. Crayton Pruitt Family Department of Biomedical Engineering, University of Florida, Gainesville, Florida 32611, ${ }^{2}$ Nathan Kline Institute for Psychiatric \\ Research, Orangeburg, New York 10962, and ${ }^{3}$ Columbia University College of Physicians and Surgeons, New York, New York 10027
}

Field potential oscillations at $\sim 10 \mathrm{~Hz}$ (alpha rhythm) are widely noted in the visual cortices, but their physiological mechanisms and significance are poorly understood. In vitro studies have implicated pyramidal neurons in both infragranular and supragranular layers as pacemakers. The generality of these observations for the intact brain in the behaving subject is unknown. We analyzed laminar profiles of spontaneous local field potentials and multiunit activity (MUA) recorded with linear array multielectrodes from visual areas V2, V4, and inferotemporal (IT) cortex of two macaque monkeys during performance of a sensory discrimination task. Current source density (CSD) analysis was combined with CSD-MUA coherence to identify intracortical alpha current generators and their potential for alpha pacemaking. The role of each alpha current generator was further delineated by Granger causality analyses. In V2 and V4, alpha current generators were found in all layers, with the infragranular generator acting as primary local pacemaking generator. In contrast, in IT, alpha current generators were found only in supragranular and infragranular layers, with the supragranular generator acting as primary local pacemaking generator. The amplitude of alpha activity in V2 and V4 was negatively correlated with behavioral performance, whereas the opposite was true in IT. The alpha rhythm in IT thus appears to differ from that in the lower-order cortices, both in terms of its underlying physiological mechanism and its behavioral correlates. This work may help to reconcile some of the diverse findings and conclusions on the functional significance of alpha band oscillations in the visual system.

Key words: alpha rhythm; laminar current generator; laminar organization; pacemaker; current source density; coherence; Granger causality

\section{Introduction}

Although 8-12 Hz oscillation (alpha rhythm) is prominent in the parieto-occipital electroencephalogram (EEG) of awake humans (Shaw, 2003; Niedermeyer, 2005), nearly 80 years after its discovery (Berger, 1929), its functional significance remains unclear. Typically, alpha rhythm is largest with the eyes closed and attenuates on eye opening and during tasks requiring visual attention (Steriade et al., 1990), leading to the notion that alpha rhythm signifies an idling state of the brain, or even a mechanism of active input suppression (Worden et al., 2000). More recent findings, however, dispute this notion, because alpha amplitude appears to increase in "rejection task" variants of working memory and mental imagery paradigms, in which attention is directed internally (Ray and Cole, 1985; Cooper et al., 2003; Palva and Palva, 2007).

The physiological mechanisms generating alpha rhythm are similarly unclear. An initial hypothesis emphasized the pacemaking role of the thalamus (Andersen and Andersson, 1968), but a series of studies in dogs (Lopes da Silva et al., 1973a,b, 1980; Lopes da Silva and van Leeuwen, 1977) reported that the alpha

Received June 11, 2008; revised July 30, 2008; accepted Aug. 21, 2008.

This work was supported by National Institutes of Health Grants MH071620, MH070498, MH079388, and MH060358. We thank A. D. Mehta and I. Ulbert for assistance in data collection.

Correspondence should be addressed to Mingzhou Ding at the above address. E-mail: mding@bme.ufl.edu. DOI:10.1523/JNEUROSCI.2699-08.2008

Copyright $\odot 2008$ Society for Neuroscience $\quad 0270-6474 / 08 / 289976-13 \$ 15.00 / 0$ rhythm is of a cortical origin with large layer 5 pyramidal neurons acting as pacemakers (Lopes da Silva, 1991). In vitro preparations have further established that cortical neurons can oscillate intrinsically (Steriade et al., 1990; Connors and Amitai, 1997), and have tested the role of layer 5 pyramidal cells in "alpha pacemaking" (Silva et al., 1991; Flint and Connors, 1996), and in "augmenting response" (Castro-Alamancos and Connors, 1996), a resonancelike phenomenon observed on repetitive stimulation at $10 \mathrm{~Hz}$. These findings have motivated computational models to incorporate an alpha pacemaker at the level of lamina 5 (Jones et al., 2000; Karameh et al., 2006). However, $10 \mathrm{~Hz}$ oscillations, which do not show the classic visual alpha reactivity, are also found in non-parieto-occipital areas such as entorhinal (Lukatch and MacIver, 1997), auditory (Lehtelä et al., 1997), and motor (CastroAlamancos and Tawara-Hirata, 2007) cortices. In the entorhinal cortex, the local alpha pacemaking role may be taken by supragranular pyramidal neurons.

To explore the generality of the in vitro findings to the intact brain (Steriade, 2004), we investigated the physiology and laminar organization of "spontaneous" alpha rhythms in occipital and temporal cortices in behaving monkeys during performance of an active auditory discrimination task. Laminar profiles of local field potential (LFP) and multiunit activity (MUA) were recorded from V2, V4, and inferotemporal cortex (IT) using linear array multielectrodes that sampled from all layers simultaneously. Laminar generators of the alpha rhythm were identified 
by phase-aligned LFP analyses, augmented by current source density (CSD) analysis. CSD-MUA coherence tested the rhythmic relationship of transmembrane current oscillations to the action potentials outputs, necessary for effective pacemaking. Coherence and Granger causality analyses (Ding et al., 2006; Le Van Quyen and Bragin, 2007) evaluated the interaction between different laminar alpha generators. Furthermore, correlation between alpha amplitude and reaction time (RT) was calculated for each area to study how alpha rhythm modulates with attention.

\section{Materials and Methods \\ Experiment}

All surgical, training, and other relevant aspects of experimental procedure were approved by the Institutional Animal Care and Use Committee.

Surgical preparation. Monkeys were prepared for chronic awake intracortical recording. Surgery was performed using aseptic techniques, under general anesthesia, as described previously (Givre et al., 1994; Schroeder et al., 1995, 1998; Chen et al., 2007; Lakatos et al., 2008). Briefly, the tissue overlying the calvarium was resected, and appropriate portions of the cranium were removed. The neocortex and overlying dura were left intact. To provide access to the brain and to promote an orderly pattern of sampling across the surface of visual areas, recording chambers incorporating parallel guide tube grids (Crist Instrument) were positioned normal to the brain surface for orthogonal penetration of (1) portions of area V2 underlying the foveal representation of area V1 in the lateral striate operculum, (2) foveal/parafoveal representations in area V4 lying on the lateral prelunate gyrus, and (3) foveal/parafoveal regions of IT cortex lying on the lower bank of the superior temporal sulcus, anterior to the MT/MST complex (medial temporal/medial superior temporal cortex) and lateral to FST (fundus of the superior temporal sulcus). Choice of these specific portions of the target regions enabled us to penetrate the target regions orthogonal to their lamination patterns to optimize the implementation of one-dimensional CSD analyses (see below). Implantation was guided by stereotaxic transformation of magnetic resonance imaging data, which delineated the cortical gyral pattern. Individual epidural guide tubes were positioned over central and frontal sites to serve as ground and reference electrodes. Together with socketed Plexiglas bars (to permit painless head restraint), they were secured to the skull with orthopedic screws and embedded in dental acrylic. A recovery time of 2 weeks was allowed before the beginning of data collection.

Paradigm. As part of an experiment involving switching attention between auditory to visual input streams, two male macaque monkeys (Macaca fascicularis), referred to as B and V, were trained to perform an auditory oddball discrimination task (Lakatos et al., 2008). In this task, 100 -ms-duration pure tones were presented at $1.5 \mathrm{~Hz}$, with the stream of "standard" stimuli randomly interrupted by tones that differed in frequency (deviants). The monkey was required to respond to the deviants after the onset of an oddball stimulus. A liquid reward was given to correct responses. The reason for analyzing activity in visual cortices during auditory discrimination was that the discrimination kept the monkey verifiably alert without using visual stimuli, so that we could study spontaneous neural activity in V2, V4, and IT cortex. In addition, the behavioral performance measured by the reaction time provided an opportunity of examining the functional significance of alpha activity in various visual cortices. Data were collected during periods of adequate task performance (i.e., $>80 \%$ target detection).

Recordings. Animals sat in a primate chair in a completely dark, electrically shielded, sound-attenuated chamber with head fixed in position, and were monitored with infrared videography. LFP and MUA were sampled $(2 \mathrm{kHz})$ with a linear array electrode with 14 contacts spanning all six cortical layers (see Fig. $1 A$ for a schematic). The electrodes were constructed by threading $25-\mu \mathrm{m}$-diameter, Teflon-coated wires through a stainless-steel tube, aligning and spacing the wires in a linear array at the distal tip of the tube (beveled), embedding the wires in fast-curing epoxy, and cutting the wires flush with the epoxy after it dried. The resulting line of recording contacts was thus $25 \mu \mathrm{m}$ in cross section, and had impedances between 0.3 and $0.5 \mathrm{M} \Omega$ at $2 \mathrm{kHz}$. The intercontact spacing used was $150 \mu \mathrm{m}$ in V2 and $200 \mu \mathrm{m}$ in V4 and IT. Multiple penetrations were made in each of the three visual cortical areas. The dataset analyzed here consists of two penetrations in V2, two penetrations in V4, and three penetrations in IT for subject B; and two penetrations in V2, two penetrations in $\mathrm{V} 4$, and one penetration in IT for subject $\mathrm{V}$.

Functional position of multielectrodes. For each experiment, electrodes were lowered into chambers targeting one or more of the aforementioned visual areas. Using the intracortical profiles of LFPs and MUA generated in response to visual stimulation, the electrodes were guided to the target region and then positioned so as to straddle its laminar expanse as described in detail previously (Schroeder et al., 1998) [see also more recent studies that have used this approach (Lipton et al., 2006; Chen et al., 2007; Lakatos et al., 2007)]. The positioning method is based on the fact that all visual areas recorded to date generate an LFP distribution with a constant amplitude decay (i.e., second derivative of approximately zero) over distance (see also Schroeder et al., 1995). Visually evoked LFP and MUA profiles are collected at successive depths beginning well in advance of the expected depth of the target region. Linear amplitude increase (near-zero second derivative) indicates approach to an active visual area. Arrival at the depth of the active region is indicated by steep acceleration in the LFP gradients and/or component polarity inversions (nonzero second derivative). MUA is also useful, because in association with a relatively flat CSD profile, active MUA indicates that the electrode array is traversing a span of white matter. Sulcal cortical visual areas are distinguished from intervening white matter regions by the conjunction of large CSD components with multiunit activity. Because the CSD indexes the net local transmembrane current flow in a cortical region, the large, current-balanced CSD profiles characteristic of cortical regions and laminated nuclear structures are not found in association with the MUA recorded in white matter. After localizing an active region, further depth adjustments are made based on the CSD profile, so as to straddle its laminar expanse with the electrode array. Because the uppermost laminae in most cortical areas are relatively quiescent, we can distinguish between sulcal areas whose pial surfaces were opposed. In this case, the relevant distinction is between IT cortex (on the lower bank of the superior temporal sulcus) and the overlying cortical regions. In any case, the outer boundaries of an active cortical region, both pial and white matter regions, are indicated by drops to near-zero CSD values. Then, after the direction from which the tissue is penetrated (pial or white matter) is determined (by track reconstruction), approximate internal laminar assignments can be made, based on the known internal geometry of the region (see below).

Localization of V2, V4, and IT. In both monkeys, visual areas were defined by anatomic location, subject to histologic verification [for detailed illustration, see Schroeder et al. (1998) and Chen et al. (2007)]. V2 recordings were made in the first belt of cortical tissue underlying the operculum, immediately posterior to the lunate sulcus, by penetrating through V1. V4 recordings were in the crown of the prelunate gyrus. IT recordings were made in the lower bank of the superior temporal sulcus.

Identification of laminae. Once the electrode is positioned so that the contacts straddle the active current flow pattern of an area, gross laminar positions can be gauged from the known anatomical dimensions of each region. In extrastriate visual cortices, for example, layer 4 lies just below the midpoint of the laminar depth of the area (Hendry et al., 1990; Jones, 1990). In addition, our previous studies have revealed some functional clues to laminar locations. In particular, we have noted that in V2, V4, and IT (other extrastriate areas as well), the largest current sink associated with the N95 component of the visual evoked potential (this corresponds to a human N1) is located at the depth of layer 4; in fact, this current sink reflects the main feedforward activation of the area from lower visual cortices (Givre et al., 1994; Schroeder et al., 1995, 1998; Chen et al., 2007).

\section{Data analysis}

The data. The neocortex is a structured tissue with high specificity of connections between its six layers (Lund, 2002). Whereas extracellular LFP, generated by transmembrane current flow, reflects mainly dendritic processing, the MUA gauges the intensity of pyramidal cell firing. Together, LFP and MUA form a comprehensive measure of cortical neuro- 
nal ensemble activity at both the input and output level (Nicholson, 1973; Mitzdorf, 1985; Schroeder et al., 1995). Previous work has shown that simultaneous recordings of LFPs and MUAs from different layers of the cortex are critical to controlled analysis of the dynamics of cortical circuitry under different behavioral states (Schroeder et al., 1995; Mehta et al., 2000a,b). It is generally accepted that the largest contributions to LFPs come from pyramidal neurons, which comprise nearly $85 \%$ of the cortex (Lopes da Silva and Rotterdam, 2005). Under certain conditions, additional neuronal elements are also known to contribute (Tenke and Schroeder, 1990; Schroeder et al., 1991, 1992, 1995). Typically, LFPs are recorded against a distant reference, making them susceptible to volume conduction of potentials from other sites. The first derivative used for generating the bipolar LFPs and the second-derivative approximation used for the CSD analysis help to eliminate this problem (Mitzdorf, 1985). Moreover, the CSD depth profile allows the localization of transmembrane current generators to specific cortical layers. Concomitant MUA helps assess the physiological processes (i.e., excitability changes) that current generators are associated with.

Logic of the analysis protocol. We seek to characterize the laminar organization of the cortical alpha rhythm by analyzing depth LFPs and MUAs. The analysis protocol has three main steps. First, transmembrane current generators that give rise to field oscillations at the alpha frequency are identified with the CSD method. Traditionally, CSD analysis is performed on field potentials averaged with respect to a repetitive sensory stimulus (Mitzdorf, 1985; Schroeder et al., 1991, 1995). The CSD of ongoing neural activity is more difficult to ascertain. Single-trial CSD estimates tend to be noisy (Shah et al., 2004; Lakatos et al., 2005, 2007), and because there is no stimulus-related trigger, LFP averaging requires an alternate procedure for the alignment of trials. The phase-realigned averaging technique (PRAT; see below) is such a procedure that was used here to identify the alpha current generators within the cortical column. Second, alpha current generators that have the potential of pacemaking are identified with CSD-MUA coherence. In the context of studying evoked potentials, a source or sink is considered active if simultaneously recorded MUA is depressed or enhanced, indexing net local hyperpolarization or depolarization, respectively. For ongoing oscillatory activity, the membrane undergoes rhythmic transition between hyperpolarization and depolarization. It is thus more appropriate to consider the oscillatory current generator in terms of its pacemaking capability. During the depolarizing phase of the oscillation, the pacemaker cells fire bursts of action potentials, which, via synaptic transmission, entrain neural activity in other laminae and cortical areas. For the present work, significant phase coherence between CSD and MUA is used to indicate that a current generator is accompanied by rhythmic firing and thus has the potential of being a pacemaker. Third, the primary local pacemaking generator is identified with the Granger causality analysis. For a cortical column with multiple alpha current generators distributed across different layers, the relationship among these generators needs to be further delineated. This is particularly so if the second step reveals that more than one generator has the potential of being the pacemaker. Granger causality analysis, based on bipolar LFPs, is used to further disambiguate the roles of different current generators, because the primary local pacemaking generator is expected to exert unidirectional causal influence on other neural ensembles (see below).

Current source density analysis of ongoing oscillatory activity. The length of a contiguous segment of spontaneous ongoing activity was on average $30 \mathrm{~s}$ long, and there were five to seven such segments for a given penetration. After high-pass filtering ( $3 \mathrm{~Hz}$, zero phase shift) and downsampling to $200 \mathrm{~Hz}$, the LFP data were further divided into epochs of $200 \mathrm{~ms}$ in duration, which were considered trials or realizations of an underlying stochastic process. To calculate the average current source density, the following procedure was used. (1) The power spectrum of each recording contact for a given electrode was estimated, and the contact showing the highest power spectral density at the alpha frequency was chosen as the "phase index contact." (2) Sinusoid of the same frequency as the spectral peak in $8-12 \mathrm{~Hz}$ was then fitted to the data from the phase index contact for each epoch to obtain the phase at that frequency with respect to the beginning of the epoch. (3) The LFP data from all the contacts were shifted according to this estimated phase to realign all the trials, and the realigned signals were then averaged across epochs (trials) to obtain the averaged LFP for each contact. (4) A second spatial derivative was calculated to produce the CSD profile (Schroeder et al., 1995), from which the alpha current generators were identified. Csicsvari et al. (2003) has used a similar method to study the oscillatory activity in the hippocampus. For ease of reference, in the rest of the paper, the above procedure will be called the PRAT, and the resulting averaged LFP and CSD are referred to as PRAT-LFP and PRAT-CSD, respectively.

CSD and MUA coherence. The MUA data were epoched the same way as the LFP data and downsampled from $2 \mathrm{kHz}$ by taking a temporal average in nonoverlapping windows of $5 \mathrm{~ms}$ duration to achieve effectively the same sampling resolution of $200 \mathrm{~Hz}$ as the downsampled LFPs. Penetrations and recording contacts with poorly recorded MUA activity were excluded from further analysis. The coherence between single-trial CSDs around alpha current generators identified by the PRAT-CSD method and the corresponding mean-centered single-trial MUAs was calculated by the multivariate autoregressive (MVAR) spectral analysis method (see below). The coherence estimates were cross-checked by standard Fourier-based routines in Matlab (MathWorks). In addition, the phase spectrum between CSD and MUA at the alpha oscillation frequency was obtained to help assess whether, during an alpha epoch, a sink (or source) corresponds with increase (or decrease) in MUA.

MVAR spectral analysis. The interaction between the current generators in different laminae was studied by coherence and Granger causality analysis. For this purpose, we made appropriate bipolar derivations of the LFPs around the alpha current generators to yield bipolar LFPs. The bipolar LFP signals were subjected to MVAR spectral analysis from which power, coherence, and Granger causality spectral estimates were derived. The essential steps of MVAR can be summarized as follows. Let $p$ channels ( $p=2$ or 3 in this study) of bipolar LFP recordings at time $t$ be denoted by $\mathbf{X}_{t}=\left(x_{1 t}, x_{2 t}, \ldots x_{p t}\right)^{\mathrm{T}}$, where T stands for matrix transposition. Assume that the data over an analysis window are described by an MVAR model:

$$
\sum_{k=0}^{m} \mathbf{A}_{k} \mathbf{X}_{t-k}=\mathbf{E}_{t}
$$

where $\mathbf{E}_{t}$ is a temporally uncorrelated residual error series with covariance matrix $\Sigma$, and $\mathbf{A}_{\boldsymbol{k}}$ are $p \times p$ coefficient matrices to be estimated from data (Ding et al., 2000). The MVAR model order $m$ was determined by the Akaike information criterion (Akaike, 1974). For the data analyzed in this study, $m=10$ (50 ms) was chosen as a tradeoff between sufficient spectral resolution and overparameterization. An adequate parametric model fit of the data means that the residual noise process $\mathbf{E}_{t}$ in Equation 1 must be temporally uncorrelated (white) (Lutkepohl, 2005). The Durbin-Watson test (Durbin and Watson, 1950) was used to check the goodness of fit. The whiteness of the residuals was confirmed at the $p=$ 0.05 significance level.

Once the model coefficients $\mathbf{A}_{\boldsymbol{k}}$ and $\Sigma$ are estimated, the spectral matrix can be evaluated as

$$
\mathbf{S}(f)=\mathbf{H}(f) \Sigma \mathbf{H}^{\star}(f),
$$

where the asterisk denotes matrix transposition and complex conjugation, and $\mathbf{H}(f)=\left(\sum_{k=0}^{m} \mathbf{A}_{k} e^{-2 \pi i k f}\right)^{-1}$ is the transfer function. The power spectrum of channel $l$ is given by $S_{l l}(f)$, which is the $l$ th diagonal element of the spectral matrix $\mathbf{S}(f)$. The coherence spectrum between channel $l$ and channel $k$ is as follows:

$$
C_{l k}(f)=\frac{\left|S_{l k}(f)\right|}{\left[S_{l l}(f) S_{k k}(f)\right]^{1 / 2}} .
$$

The value of coherence ranges from 1 to 0 , with 1 indicating maximum interdependence between channel $l$ and channel $k$ at frequency $f$ and 0 indicating no interdependence. For $p=2$ in Equation 1, the Granger causality spectrum from $x_{2 t}$ to $x_{1 t}$ is defined as follows (Geweke, 1982; Brovelli et al., 2004): 


$$
I_{2 \rightarrow 1}(f)=-\ln \left(1-\frac{\left(\sum_{22}-\frac{\sum_{12}^{2}}{\sum_{11}}\right)\left|H_{12}(f)\right|^{2}}{S_{11}(f)}\right),
$$

which can be interpreted as the proportion of $x_{2 t}$ 's causal contribution to the power of the $x_{1 t}$ series at frequency $f$. The logarithm is taken to preserve certain favorable statistical properties. Similarly, the causality spectrum from $x_{1 t}$ and $x_{2 t}$ can be obtained by switching the indices 1 and 2 in Equation 4. In addition to the bivariate causality algorithm, we also applied a conditional Granger causality algorithm (Chen et al., 2006; Ding et al., 2006), which allows the examination of whether the causal influence between two time series is direct or is mediated by a third time series.

Interpretation of Granger causality. For two simultaneously measured time series, one series can be called causal to the other if we can better predict the second series by incorporating past knowledge of the first one (Wiener, 1956). This concept was later adopted and formalized by Granger (1969) in the context of linear regression models of stochastic processes (see Eq. 1). Specifically, if the variance of the prediction error for the second time series at the present time is reduced by including past measurements from the first time series in the linear regression model, then the first time series can be said to have a causal (directional or driving) influence on the second time series. Reversing the roles of the two time series, one repeats the process to address the question of causal influence in the opposite direction. From this definition, it is clear that the flow of time plays an essential role in allowing inferences to be made about directional causal influences from time series data. Alternatively, improvement in prediction can also be viewed from the perspective of comparing relative estimates of conditional probability. Recent work has modeled the relation between multiple point processes along this view (Okatan et al., 2005; Truccolo et al., 2005). In our analysis, the bipolar LFP data constitute the time series, and Granger causal influence is equated with the direction of synaptic transmission between neuronal ensembles. Physiologically, rhythmic synaptic activity (LFP) in a neuronal ensemble gives rise to periodic bursts of action potential firing (MUA), which, through synaptic transmission, drives the rhythmic synaptic activity in the target neuronal ensemble. Thus, evaluation of the causal relation between two bipolar LFP time series forms the basis for identifying the layer containing the pacemaking cells in a column. In this study, Granger causality analysis, as a principled approach for inferring causal influence among neural time series (Freiwald et al., 1999; Bernasconi et al., 2000; Liang et al., 2000; Baccalá and Sameshima, 2001; Hesse et al., 2003; Brovelli et al., 2004; Ding et al., 2006), is used in lieu of the trisection method in in vitro studies to identify the laminar pacemakers of the alpha rhythm. We analyzed bipolar LFPs rather then simple LFPs because the latter are vulnerable to contamination by volume conduction. The use of bipolar LFPs rather than CSDs was based on the consideration that the latter are more noisy in single trials (Lakatos et al., 2005, 2007; Shah et al., 2004), and excessive noise can lead to spurious causality results (Nalatore et al., 2007).

Assessment of statistical significance. For coherence and Granger causality spectra, we have adopted a random permutation approach (Brovelli et al., 2004) to build a baseline null-hypothesis distribution from which statistical significance can be derived. Consider two channels of recordings with many repeated trials (one trial being a $200 \mathrm{~ms}$ epoch for this work). We can reasonably assume that the data from different trials are approximately independent of one another. Randomly pairing data for channel 1 with data for channel 2 from a different trial leads to the creation of a synthetic ensemble of trials for which there is no interdependence between the two channels by construction. However, the temporal structure within a channel is preserved. Performing such random pairing with many different permutations will result in a distribution of coherence or causality spectra corresponding to the null hypothesis of no statistical interdependence. The calculated value for a given statistic from the actual data is compared with this baseline null-hypothesis distribution for the assessment of significance levels. In addition, the difference between two nonzero quantities was tested by a bootstrap resampling technique (Efron, 1982).

Visual alpha power and auditory reaction time. The functional signifi- cance of alpha activity in visual cortices was assessed by correlating its magnitude with auditory behavioral performance. For each $30 \mathrm{~s} \mathrm{segment}$ of contiguous data analyzed (five to seven such segments in a penetration), the monkey made on average six lever-pressing responses to the deviant auditory stimulus (see Experiment, Paradigm). With a target detection rate of $\sim 90 \%$, there were 25-35 correct responses for each penetration. For each correct response, an $800 \mathrm{~ms}$ interval before the onset of a deviant stimulus was defined as the prestimulus interval. All the correct responses in a penetration were rank ordered by the reaction time and then sorted into groups of five, starting with the fastest reaction times and proceeding to the slowest, each group sharing four trials with the previous one. For each group, the alpha power was estimated for each electrode contact and averaged across all 14 electrode contacts. The relation between the average group reaction time and average group alpha power was assessed by Spearman's rank correlation. The same alpha power and reaction time relation was also examined on a contact-bycontact basis.

\section{Results}

Two macaque monkeys participated in the study. Seated in a chair in a dark room, the monkey performed an auditory discrimination task (see Materials and Methods). The performance accuracy (i.e., target detection) was $>85 \%$, demonstrating that the subjects were alert, a state in which the alpha rhythm is known to be prominent. During task performance, laminar profiles of LFP and MUA activities were recorded with a linear multielectrode in visual areas V4, V2, and IT. Four penetrations in each area in which neural activity was reliably sampled from all layers of the cortex were selected for further analysis. Each penetration contained 150-210 s of spontaneous activity. Robust alpha oscillations could be readily seen in LFP recordings from all areas. The power spectrum averaged across the 14 recording contacts in the multielectrode revealed peak frequency of $10.1 \pm 0.8 \mathrm{~Hz}$ in V4, $9.8 \pm 1.2 \mathrm{~Hz}$ in $\mathrm{V} 2$, and $10.4 \pm 1.8 \mathrm{~Hz}$ in IT.

\section{Laminar distribution of alpha generators in V4}

Figure $1 B$ depicts a single $200 \mathrm{~ms}$ epoch of LFP data from V4 in which two full cycles of a rhythmic oscillation at $10 \mathrm{~Hz}$ are apparent. Note that each cycle undergoes polarity inversion in the middle cortical layers, and their amplitudes appear largest in the infragranular (IG) layers (deep layers). Spectral power at $10 \mathrm{~Hz}$ computed over all epochs of LFP data confirmed this impression; as shown in Figure $1 D$, alpha power in the IG layers is consistently higher than in the granular $(G)$ (layer 4) and supragranular (SG) layers (superficial layers) for all four penetrations in both monkeys. To generate the $10 \mathrm{~Hz}$ CSD profile, we applied the PRAT method by selecting the contact with the highest spectral power as the phase index contact (contact 12 for Fig. 1). The resulting PRAT-LFP and PRAT-CSD profiles derived from it are superimposed in Figure $1 C$, on the time scale of one cycle of the $10 \mathrm{~Hz}$ oscillation. Overlain on these profiles is a single epoch of MUA signals from a subset of the recording contacts. Current sources (blue) and sinks (red) underlying the generation of the oscillatory alpha field activity are readily identified in G, IG, and SG layers, where the current sinks (red) correspond to negative PRAT-LFPs. These characteristics in the laminar PRAT-CSD profile in Figure $1 C$ were found to be consistent across all four penetrations in both monkeys (supplemental Fig. 1, available at www.jneurosci.org as supplemental material). As noted by Csicsvari et al. (2003), the choice of the phase index contact biases the CSD toward detecting neural activity close to that electrode contact. We found that using a different contact, for example channel 6 in the above dataset, resulted in a strengthening of alpha currents in $\mathrm{G}$ and SG layers and a weakening in the IG layers, but did not change the overall CSD profile. 
The increases in MUA accompanying the middle and lower layer sinks in the latter half of the $10 \mathrm{~Hz}$ cycle displayed in Figure $1 C$ suggest that these sinks reflect net depolarization of the local neurons, and concomitant MUA decreases suggest that the reverse is true for the colocated current sources (blue) in the initial part of the cycle. The early and late parts of the alpha cycle thus appear to represent the low- and high-excitability phases of the local alpha oscillation. To assess more quantitatively the extent to which the firing probability of the local neurons around a given current generator is phase locked to the alpha current oscillation, we estimated the coherence between CSD and MUA for each alpha current generator. The coherence spectra have clear peaks at $\sim 10 \mathrm{~Hz}$ in the IG and $G$ layers as shown in Figure $1 E$. The peak coherence is $0.18(p<0.01)$ in IG layers, and $0.16(p<0.01)$ in G layer, suggesting that the neuronal firing at these generators is phase locked to the underlying oscillatory current. In contrast, the CSD-MUA coherence for the SG layer did not show an alpha peak (Fig. $1 E$ ), and the coherence value at $10 \mathrm{~Hz}$ was not significant $(0.002 ; p=0.23)$. Note that the SG current generator is out of phase with that in $\mathrm{G}$ and IG layers. Thus, a plausible explanation for the lack of significant CSDMUA coherence in the SG layers is dampening caused by inhibition (Schroeder et al., 1995).

The CSD-MUA relationship can be further quantified by computing their relative phase at $10 \mathrm{~Hz}$ to help determine whether increase in firing in a local group of neurons around a given current generator corresponds to a sink and, thus, reflects net local neuronal depolarization. The phase spectrum (data not shown) was found to be continuous in the alpha range $(8-12 \mathrm{~Hz})$ with a mean phase difference of $169.0 \pm 3.4^{\circ}$ and $156.4 \pm 3.3^{\circ}(n=3$ penetrations; with CSD leading) for G and IG layers, respectively. This nearly out-ofphase relationship between CSD and MUA implies that the increased action potential firing in IG and $G$ layers coincide with the alpha current sinks.

The CSD-MUA coherence characteristics were consistent across three penetrations from two monkeys. The fourth penetration showed little phasic variation in MUA after visual stimulus presentation, and was excluded from the CSD-MUA coherence analysis. Thus, the consistent biasing of the CSD-MUA coherence toward the G and the IG layers (Fig. $1 E$ ), together with the laminar distribution of alpha power in Figure $1 D$, strongly suggests that the neural ensembles in the $G$ and IG layers are potential local alpha pacemakers. The more precise relationship between these potential pacemakers is examined by a Granger causality analysis below. A
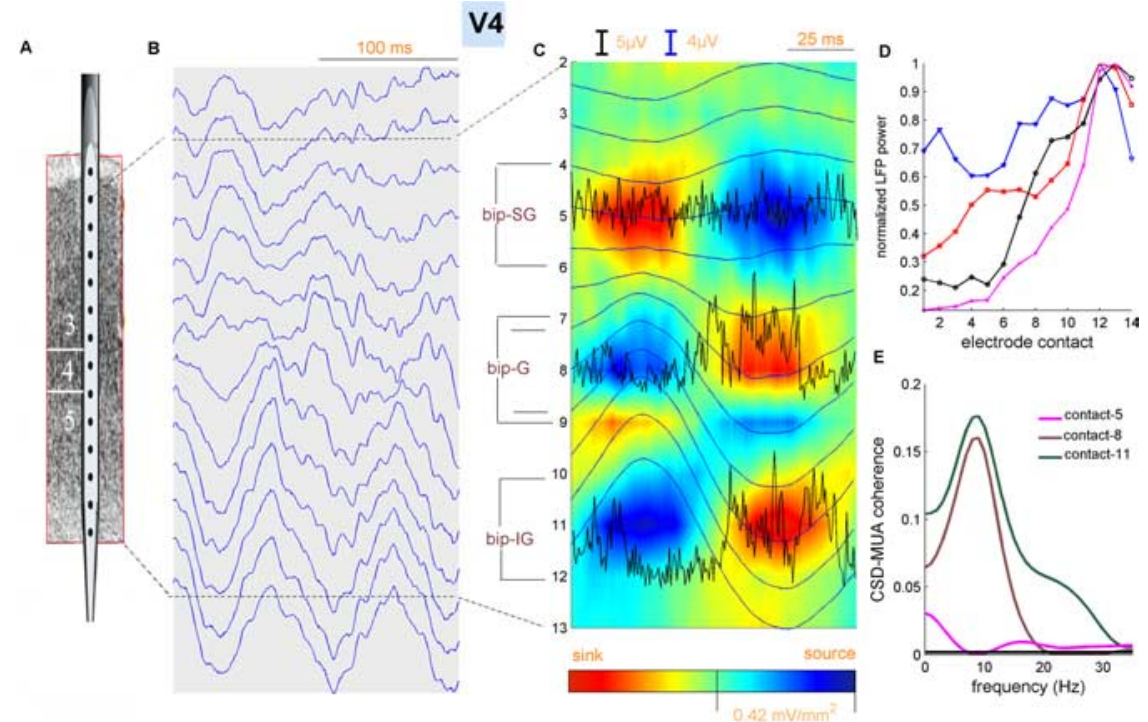

Figure 1. Results for V4. $\boldsymbol{A}$, Schematic of the multielectrode with 14 equally spaced (200 $\mu \mathrm{m})$ contacts. $\boldsymbol{B}$, A short segment (200 ms) of LFPs. C, PRAT-CSD displayed as a color-coded plot, which is the second spatial derivative of phase-realigned and averaged PRAT-LFPs (smooth traces, blue). The $y$-axis is electrode contacts from 2 to 13. The contacts used for bipolar (bip) derivations are shown to the left (see Fig. 4 and Results, Interaction of alpha current generators). A single epoch of MUA from three contacts is superimposed (black). $\boldsymbol{D}$, Laminar distribution of the peak $(10 \mathrm{~Hz})$ LFP power across all penetrations in both monkeys. $\boldsymbol{E}$, CSD-MUA coherence spectra for the penetration shown in $\boldsymbol{C}$. The horizontal line corresponds to $p=0.01$.
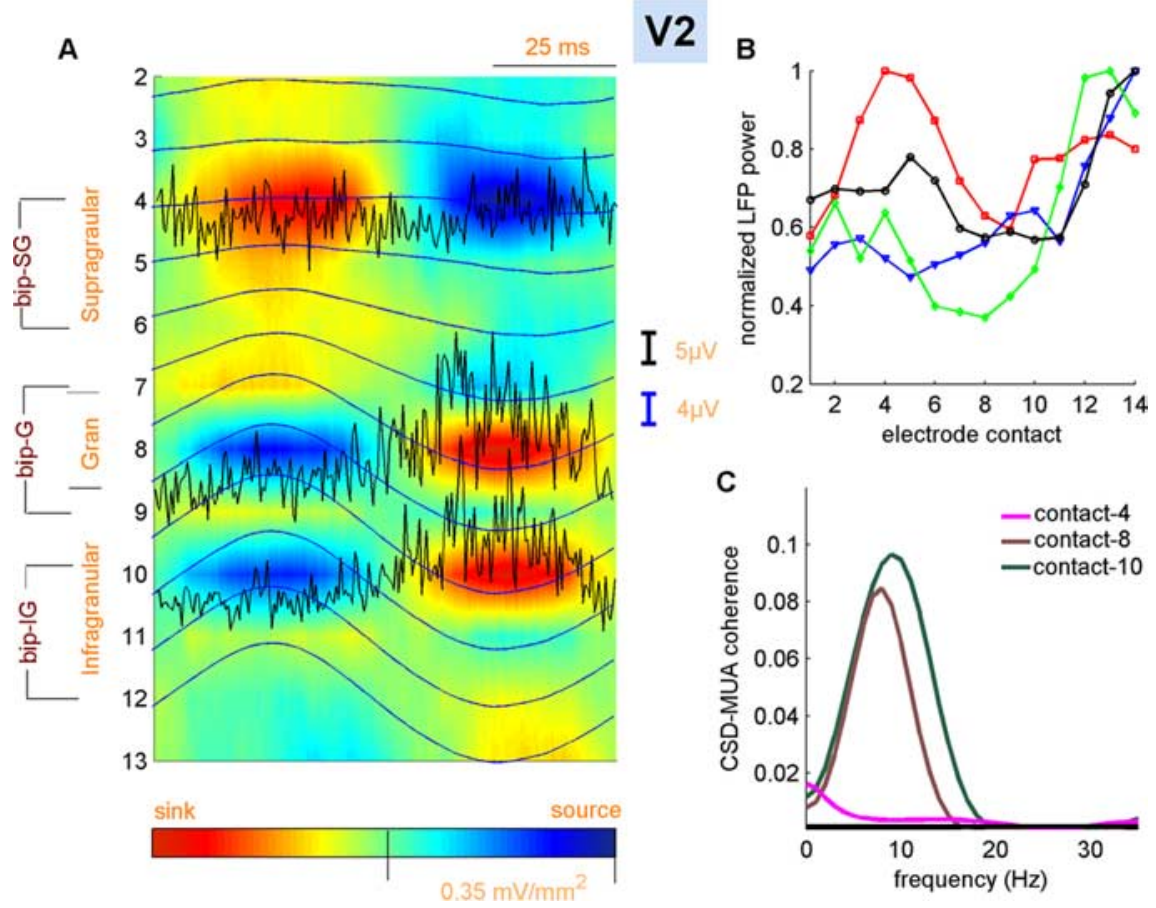

Figure 2. Results for V2. Gran, Granular. Conventions are otherwise the same as in Figure 1.

unidirectional causal influence at $10 \mathrm{~Hz}$ from one potential pacemaker to another would establish the former as the primary local alpha pacemaker.

\section{Laminar distribution of alpha generators in V2}

The PRAT-LFP and PRAT-CSD profiles from a representative penetration in V2, together with a single epoch of MUA signals, are shown in Figure $2 A$; here, contact 12 was used as the phase index contact. Temporal alternations of current sources (blue) 


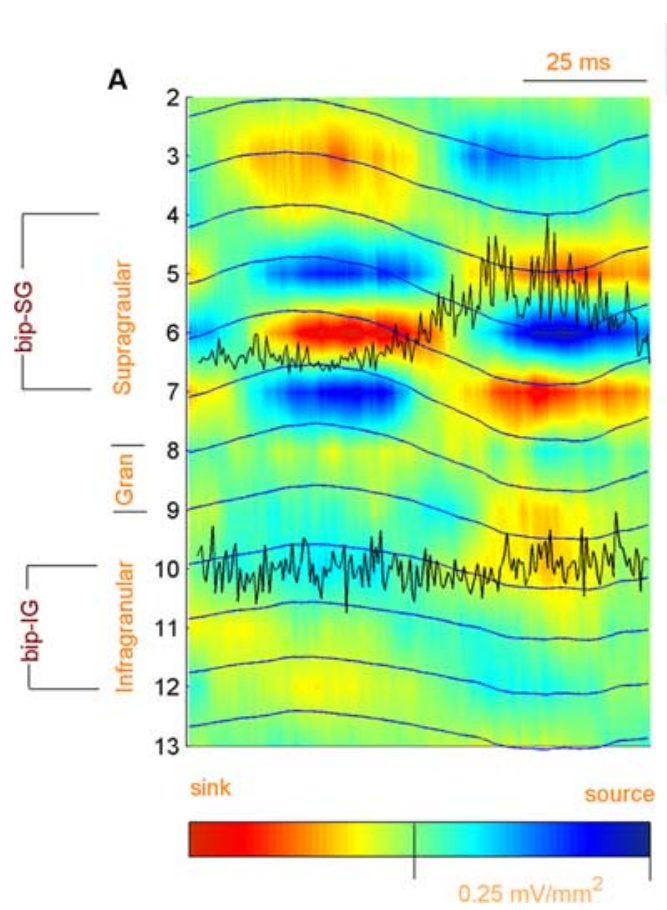

Figure 3. Results for IT. cont., Contact; Gran, granular. Conventions are otherwise the same as in Figure 1.
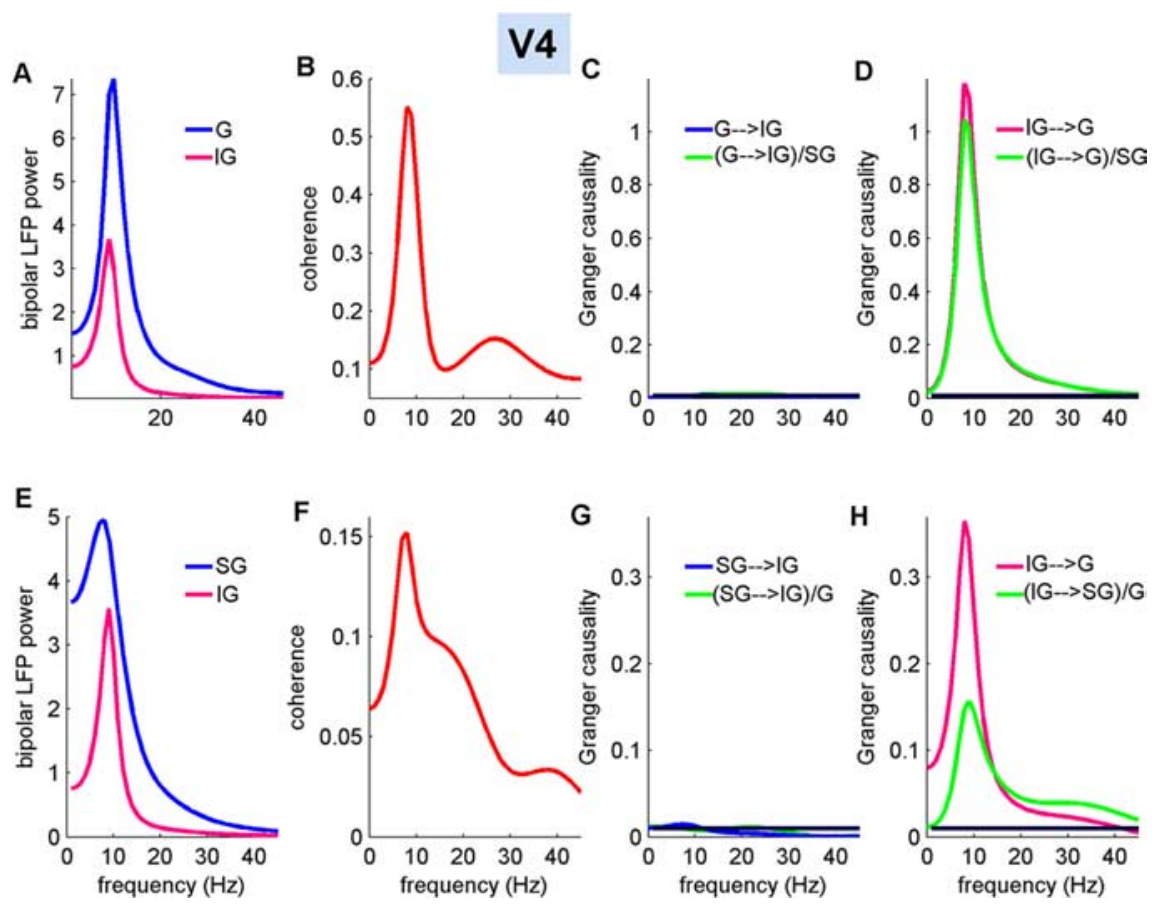

Figure 4. MVAR spectral analysis for the V4 penetration shown in Figure $1 C$. $A$, Power spectra of bipolar LFP signals at $G$ and IG layers. $\boldsymbol{B}$, Coherence spectrum between the two bipolar signals in $\boldsymbol{A}$. C, $\boldsymbol{D}$, Granger causality spectra between $G$ and IG. Here, $x \rightarrow y$ denotes $x$ driving $y$, and $(x \rightarrow y) / z$ denotes $x$ driving $y$ after conditioning out $z$. E, Power spectra of the bipolar LFP signals at $S G$ and IG layers. $\boldsymbol{F}$, Coherence spectrum between the two bipolar signals in $\boldsymbol{E}$. $\boldsymbol{G}, \boldsymbol{H}$, Granger causality spectra between $\mathbf{S}$ and IG. The horizontal lines in $\mathbf{C}, \boldsymbol{D}, \mathbf{G}$, and $\boldsymbol{H}$ correspond to $p=0.01$.

and sinks (red) occurring at the alpha frequency can be seen in IG, G, and SG layers. These laminar characteristics were present in all four V2 penetrations (supplemental Fig. 2, available at www.jneurosci.org as supplemental material). In addition, the similarity between the PRAT-CSD profile in V2 and that in V4 is readily identifiable. The distribution of the $10 \mathrm{~Hz}$ power across

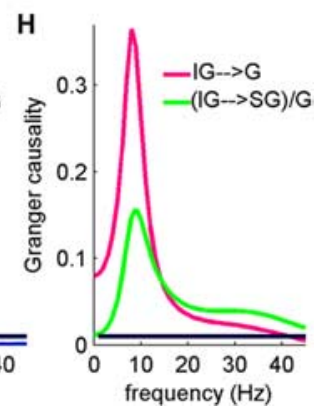

different layers exhibits increased variability compared with V4, although the IG layers still have the highest power in three out of four penetrations (Fig. 2B). For one penetration, the highest alpha power occurs in the SG layers.

The CSD-MUA coherence spectra in V2 shown in Figure $2 C$ are also similar to those in V4 (Fig. 1E). The bias is again toward the lower cortical layers. The peak coherence $(9 \mathrm{~Hz})$ in IG and $(8 \mathrm{~Hz})$ G layers are found to be $0.096(p<0.01)$ and 0.082 $(p<0.01)$, respectively. The coherence spectrum in SG layers does not have an alpha peak and was barely significant at $p=0.05$ threshold level. The phase spectrum (data not shown) is found to be continuous in the alpha range $(8-12 \mathrm{~Hz})$, with a mean phase difference in $G$ and IG layers equal to $172.9 \pm 5.0^{\circ}$ and $148.9 \pm 5.0^{\circ}$ ( $n=2$ penetrations in one animal; with CSD leading), respectively. The out-ofphase relationship between MUA and CSD suggests that increased firing activity in IG and G layers coincide with current sinks generated by net depolarization of the local neurons, as illustrated by the MUA overlain with the LFP and CSD profiles in Figure $2 B$. Based on the relationship of MUA changes with the local current sink and source distributions, the early and late portions of the $10 \mathrm{~Hz}$ cycle represent the low- and high-excitability phases of the local alpha oscillation, respectively. The CSD-MUA coherence characteristics are consistent in two penetrations in one monkey. The two penetrations in the other monkey did not show clear evoked MUA on the presentation of a visual stimulus and were not included in the CSD-MUA coherence analysis.

\section{Laminar distribution of alpha generators in IT}

Figure $3 A$ shows the PRAT-LFP and PRAT-CSD profiles from a representative penetration, where the phase index contact was contact 6 . A comparison with Figures 1 and 2 revealed a number of qualitative differences. First, the alpha current generator in the SG layers (around contacts $5-7$ ) is strong and has an underlying source/sink/ source configuration. Second, superficial to the SG layer generator, a relatively strong alpha current generator is seen around contact 3 . Third, the alpha current generator in the IG layers (around contact 10 ) is weak. Fourth, no alpha current is seen in the granular layer. These laminar characteristics are generally consistent across all four penetrations in both monkeys (supplemental Fig. 3, available at www.jneurosci.org as supplemental material). The strength of the IG layer generator is very weak except for one penetration. Also in contrast to V2 and V4, the $10 \mathrm{~Hz}$ LFP power 
Table 1. Peak coherence and Granger causality values in alpha range across all penetrations for area V4

\begin{tabular}{|c|c|c|c|c|c|c|c|c|}
\hline \multirow[b]{2}{*}{ Penetration } & \multirow{2}{*}{$\frac{\text { Coherence }}{\mathrm{IG \leftrightarrow G}}$} & \multicolumn{3}{|c|}{ Granger causality } & \multirow{2}{*}{$\frac{\text { Coherence }}{I G \leftrightarrow S G}$} & \multicolumn{3}{|c|}{ Granger causality } \\
\hline & & $\mathrm{IG} \rightarrow \mathrm{G}$ & $\mathrm{G} \rightarrow \mathrm{IG}$ & $(\mathrm{IG} \rightarrow \mathrm{G}) / \mathrm{SG}$ & & $\mathrm{IG} \rightarrow \mathrm{SG}$ & $\mathrm{SG} \rightarrow \mathrm{IG}$ & $(\mathrm{IG} \rightarrow \mathrm{SG}) / \mathrm{G}$ \\
\hline \multicolumn{9}{|l|}{ B1 } \\
\hline$f(\mathrm{~Hz})$ & 9 & 9 & - & 9 & 8 & 8 & - & 9 \\
\hline Peak & 0.55 & 1.18 & NS & 1.04 & 0.15 & 0.36 & NS & 0.15 \\
\hline \multicolumn{9}{|l|}{ B2 } \\
\hline$f(\mathrm{~Hz})$ & 8 & 10 & 10 & 10 & 8 & 9 & 8 & 9 \\
\hline Peak & 0.42 & 0.84 & 0.03 & 0.73 & 0.27 & 0.23 & 0.03 & 0.09 \\
\hline \multicolumn{9}{|l|}{ V1 } \\
\hline$f(\mathrm{~Hz})$ & 9 & 10 & 10 & 10 & 9 & 9 & - & 10 \\
\hline Peak & 0.60 & 0.57 & 0.06 & 0.51 & 0.38 & 0.55 & NS & 0.24 \\
\hline \multicolumn{9}{|l|}{ V2 } \\
\hline$f(\mathrm{~Hz})$ & 10 & 10 & - & 10 & 8 & 8 & - & 8 \\
\hline Peak & 0.29 & 0.44 & NS & 0.40 & 0.13 & 0.12 & NS & 0.05 \\
\hline
\end{tabular}

Peak values are displayed together with the peak frequency in hertz. Values are only shown for spectra determined to be significant by the permutation procedure described in Materials and Methods. Here, $x \rightarrow y$ denotes $x$ driving $y$ and $(x \rightarrow y) / z$ denotes $x$ driving $y$ after conditioning out $z$. - Redundant.

in IT is generally higher in the SG layers than in the IG and G layers (Fig. 3B). Like alpha power, CSD-MUA coherence shows a supragranular bias (Fig. 3C). The peak $(10 \mathrm{~Hz})$ coherence values at contacts 5-7 are found to be $0.16(p<0.01), 0.16(p<$ $0.01)$, and $0.12(p<0.01)$, respectively. The CSD-MUA coherence at the weak current generator in the IG layers and a contact in G layer (contact 9), where no alpha current generator was found, is not significant at $p=0.05$ level (Fig. 3C). These CSD-MUA coherence results are consistent in two penetrations for which quality MUA data were recorded. The MUAs from the remaining two penetrations did not show clear phasic change after visual stimulus presentation and were thus excluded from CSD-MUA coherence analysis.

The source/sink/source configuration for the alpha current generator in the SG layers most likely reflects synaptic activity of the superficial pyramidal neuron ensemble. The CSD-MUA analysis suggests that this neuron ensemble is the likely candidate for local alpha pacemaking. Anatomically, the size of the basal dendritic arbor of the superficial pyramidal neurons has been shown to increase along the occipitotemporal hierarchy of the macaque monkey, with the arbor diameter reaching dimensions of $\sim 400 \mu \mathrm{m}$ (Elston et al., 1999) in IT. Additionally, the sinks at contacts 5 and 7 in Figure $3 A$ correspond to the largest negative deflections in the PRAT-LFPs (overlaid on PRAT-CSD in Fig. 3A). The mean phase differences in alpha range between CSD and MUA at contacts 5-7 were found to be $166.8 \pm 3.4^{\circ}$, $-38.7 \pm 4.4^{\circ}$, and $174.8 \pm 3.72^{\circ}(n=2$ penetrations; CSD leading), respectively. These results, along with concomitant MUA in Figure $3 A$ and the systematic CSD-MUA coherence analysis, indicate that the observed CSD pattern is likely the result of basal dendritic excitation where increased firing coincided with sinks at the basal dendrites. Thus, the early part of the alpha cycle displayed in Figure $3 A$ represents the low-excitability phase of the local alpha oscillation, whereas the later half of opposite sign activity represents its high-excitability phase. The current generator around contact 3 , which is out of phase with the basal den-
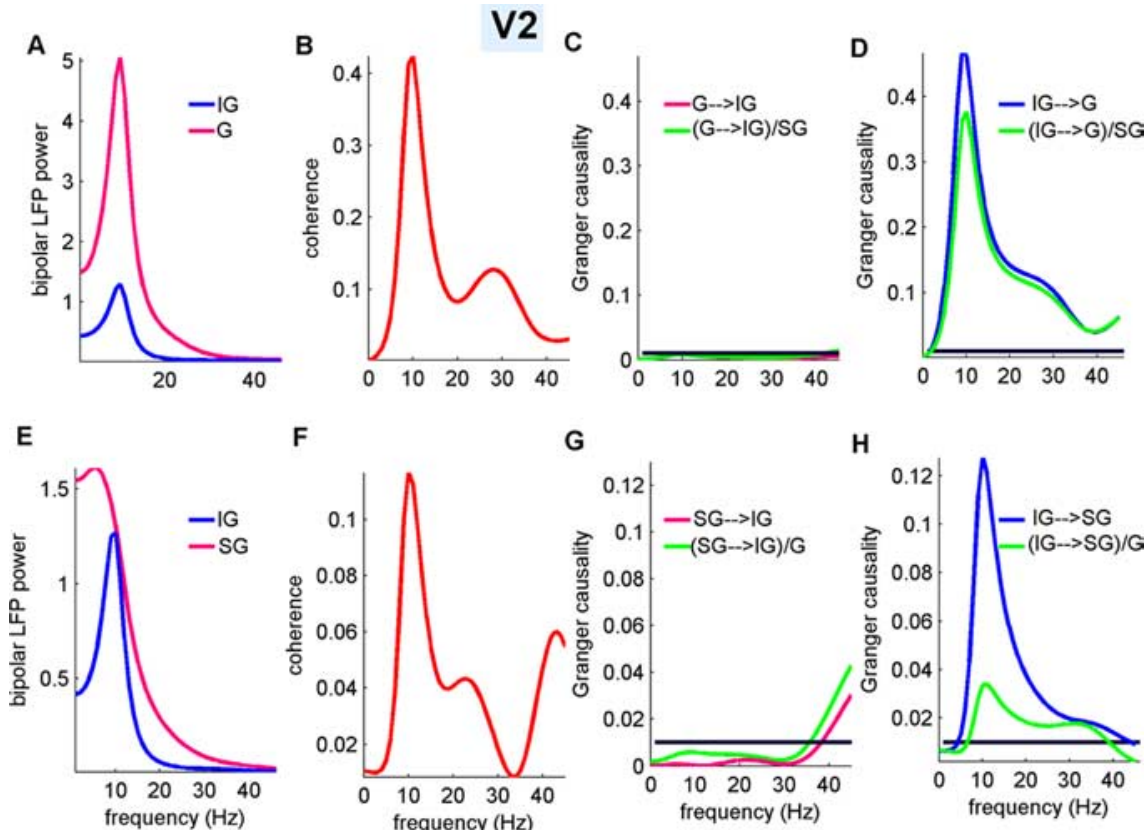

$\mathbf{F}$

G
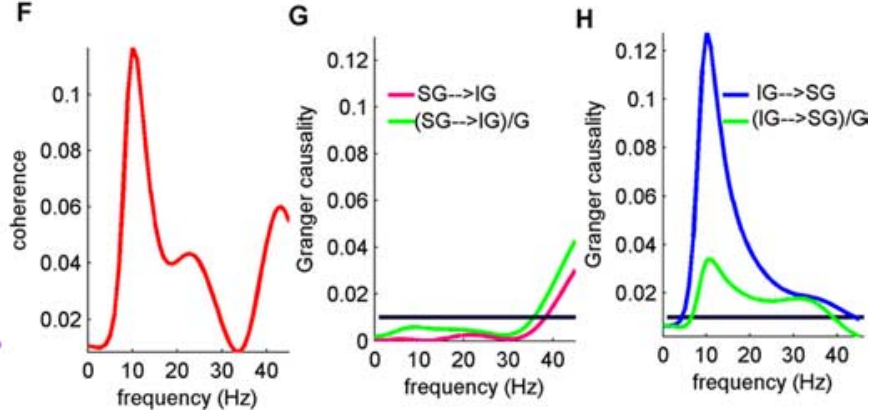

Figure 5. MVAR spectral analysis for the V2 penetration shown in Figure 2A. Conventions are otherwise the same as in Figure 4.

dritic excitation, may reflect return current through the apical dendrites.

\section{Interaction of alpha current generators}

The above analysis is largely descriptive, and only partially addressed the relationship among different laminar alpha current generators. In particular, both the granular and infragranular layer generators remain viable candidates for alpha pacemaking in V2 and V4. To explore this issue further, we performed a Granger causality analysis using appropriately derived bipolar signal to represent local neural activity around each current generator. For the V4 penetration in Figure 1, the three bipolar signals are as follows: $\mathrm{SG}=\mathrm{LFP}($ contact 6$)-\mathrm{LFP}($ contact 4$), \mathrm{G}=$ $\mathrm{LFP}($ contact 9) $-\mathrm{LFP}($ contact 7$)$, and IG $=\mathrm{LFP}($ contact 12$)-$ $\mathrm{LFP}$ (contact 10). For the V2 penetration in Figure 2 we have $\mathrm{SG}=\mathrm{LFP}($ contact 6$)-\operatorname{LFP}($ contact 4$), \mathrm{G}=\mathrm{LFP}($ contact 9$)-$ $\operatorname{LFP}($ contact 7$)$, and IG $=\operatorname{LFP}($ contact 12$)-\operatorname{LFP}($ contact 10$)$. For the IT penetration in Figure 3, the two bipolar derivations are as follows: $\mathrm{SG}=\mathrm{LFP}($ contact 7$)-\mathrm{LFP}($ contact 4$)$ and IG $=\mathrm{LFP}$ (contact 12) - LFP (contact 10). The bipolar treatment removes 
Table 2. Peak coherence and Granger causality values across all penetrations for area V2

\begin{tabular}{|c|c|c|c|c|c|c|c|c|}
\hline \multirow[b]{2}{*}{ Penetration } & \multirow{2}{*}{$\frac{\text { Coherence }}{\mathrm{IG \leftrightarrow G}}$} & \multicolumn{3}{|c|}{ Granger causality } & \multirow{2}{*}{$\frac{\text { Coherence }}{\mathrm{IG \leftrightarrow SG}}$} & \multicolumn{3}{|c|}{ Granger causality } \\
\hline & & $\mathrm{IG} \rightarrow \mathrm{G}$ & $\mathrm{G} \rightarrow \mathrm{IG}$ & $(\mathrm{IG} \rightarrow \mathrm{G}) / \mathrm{SG}$ & & $\mathrm{IG} \rightarrow \mathrm{SG}$ & $S G \rightarrow I G$ & $(I G \rightarrow S G) / G$ \\
\hline \multicolumn{9}{|l|}{ B1 } \\
\hline$f(\mathrm{~Hz})$ & 10 & 9 & - & 10 & 10 & 10 & - & 10 \\
\hline Peak & 0.42 & 0.46 & NS & 0.37 & 0.11 & 0.12 & NS & 0.03 \\
\hline \multicolumn{9}{|l|}{ B2 } \\
\hline$f(\mathrm{~Hz})$ & 11 & 9 & 9 & 9 & 10 & 10 & - & 10 \\
\hline Peak & 0.36 & 0.40 & 0.05 & 0.40 & 0.12 & 0.14 & NS & 0.05 \\
\hline \multicolumn{9}{|l|}{ V1 } \\
\hline$f(\mathrm{~Hz})$ & 7 & 8 & - & 8 & 7 & 8 & 8 & 7 \\
\hline Peak & 0.67 & 0.65 & NS & 0.58 & 0.60 & 0.52 & 0.03 & 0.30 \\
\hline \multicolumn{9}{|l|}{ V2 } \\
\hline$f(\mathrm{~Hz})$ & 7 & 9 & 8 & 9 & 7 & 8 & 8 & 8 \\
\hline Peak & 0.53 & 0.49 & 0.03 & 0.49 & 0.33 & 0.23 & 0.10 & 0.22 \\
\hline
\end{tabular}

The conventions are the same as in Table 1.

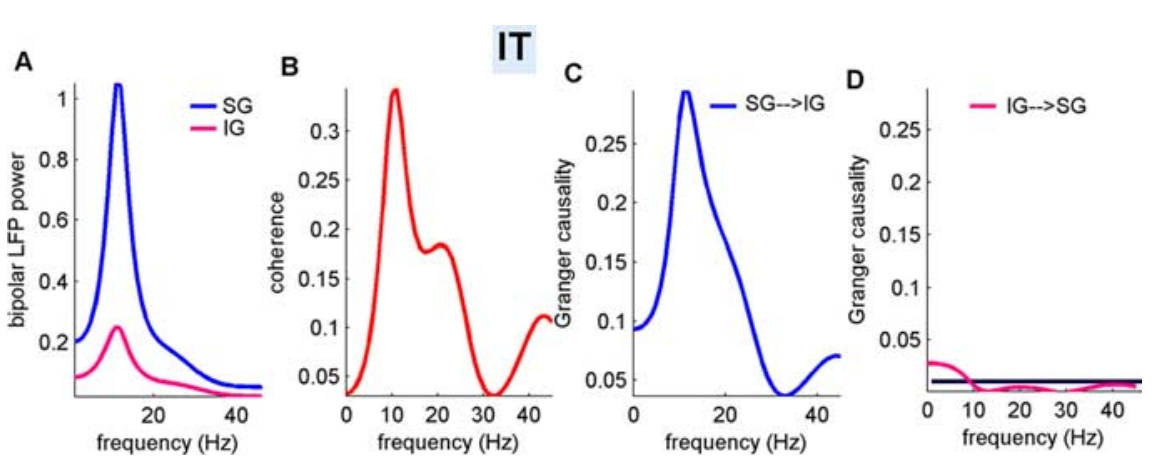

Figure 6. MVAR spectral analysis for the IT penetration shown in Figure $3 A$. Conventions are otherwise the same as in Figure 4.

Table 3. Peak coherence and Granger causality values across all penetrations for area IT

\begin{tabular}{|c|c|c|c|}
\hline \multirow[b]{2}{*}{ Penetration } & \multirow{2}{*}{$\frac{\text { Coherence }}{\mathrm{IG \leftrightarrow G}}$} & \multicolumn{2}{|c|}{ Granger causality } \\
\hline & & $\mathrm{SG} \rightarrow \mathrm{IG}$ & $\mathrm{IG} \rightarrow \mathrm{SG}$ \\
\hline \multicolumn{4}{|l|}{ B1 } \\
\hline$f(\mathrm{~Hz})$ & 11 & 11 & - \\
\hline Peak & 0.34 & 0.29 & NS \\
\hline \multicolumn{4}{|l|}{ B2 } \\
\hline$f(\mathrm{~Hz})$ & 10 & 10 & 10 \\
\hline Peak & 0.56 & 0.13 & 0.03 \\
\hline \multicolumn{4}{|l|}{ B3 } \\
\hline$f(\mathrm{~Hz})$ & 8 & 9 & - \\
\hline Peak & 0.59 & 0.23 & NS \\
\hline \multicolumn{4}{|l|}{ V1 } \\
\hline$f(\mathrm{~Hz})$ & 10 & 9 & 10 \\
\hline Peak & 0.43 & 0.39 & 0.04 \\
\hline
\end{tabular}

the effect of far fields and the common reference, and yields signals that were analyzed by bivariate (V2, V4, and IT) and conditional Granger causality (V2 and V4) measures, in addition to power and coherence. It is worth noting that the bipolar treatment may distort power distribution across different layers (compare Figs. 1D, 4A). In particular, if the power is more uniform across various contacts within a layer, taking a difference between different contacts will give a smaller signal, even though the original signal might be large. However, the coherence and Granger causality analysis is not affected by this, because they are normalized quantities and only assess the degree of dependence between different signals.

In V4, for IG and $\mathrm{G}$ layers, the bipolar LFP power spectra exhibit clear peaks at $\sim 10 \mathrm{~Hz}$ (Fig. $4 A$ ). The coherence spectrum has a pronounced peak at $9 \mathrm{~Hz}$, where the peak value is $0.55(p<0.001)$, as shown in Figure $4 B$. This suggests that the alpha currents in these layers are highly synchronized. The Granger causality spectrum of IG $\rightarrow$ G (Fig. 4D) shows a strong peak at 9 $\mathrm{Hz}$ with a peak value $1.18(p<0.001)$, whereas the causality in the other direction $(\mathrm{G} \rightarrow \mathrm{IG})$ is not significant (Fig. $4 C$ ), indicating that neural activity in the $G$ layer is strongly driven by that in the IG layers. The strong $\mathrm{IG} \rightarrow \mathrm{G}$ layer causal influence is found for all four penetrations in both monkeys (Table 1), whereas the G layer shows a weak but significant causal influence on the IG layer in two penetrations (Table 1). To examine the influence of the SG layers on the interaction between the G and IG layers, we included the bipolar LFP signal from the SG layer and performed conditional Granger causality analysis. The Granger causality from IG to G layer after conditioning out SG layer activity is nearly identical to the bivariate case (Fig. $4 D$ ), except for a small decrease in peak value. To test whether the small decrease is significant, we compared the bootstrap resampled distribution of the peak Granger causality values from the spectrum of the bivariate analysis with the peak conditional causality spectral analysis. For the penetration shown in Figure 4, the small decrease in peak value $(1.18-1.04)$ is significant $(p<0.05)$, but for all other penetrations, the decrease is found to be not significant at $p=0.05$ significance level, suggesting that the SG layers has no influence on the interaction between the IG and G layers. This is an expected result, because the CSD-MUA coherence analysis has already demonstrated that the SG layer alpha current generator is not accompanied by rhythmic firing and thus not capable of pacemaking.

The interaction between the IG and SG layers was studied by first performing a bivariate analysis. Figure $4, E$ and $F$, shows the power and coherence spectra, respectively. The power of the bipolar LFP signal for the SG layer has a clear peak at $8 \mathrm{~Hz}$. The coherence spectrum also peaks at $8 \mathrm{~Hz}$ with a peak value of 0.15 $(p<0.001)$, indicating a significant (but weak) synchrony between the local alpha currents in these two layers. Granger causality again reveals IG as the driver of the SG current with the peak value of $0.36(p<0.001)$ at $8 \mathrm{~Hz}($ Fig. $4 H)$. The causal influence in the opposite direction ( $\mathrm{SG} \rightarrow \mathrm{IG}$ ) is not significant (Fig. $4 G$ ). Significant $I G \rightarrow S G$ is found in all four penetrations in both monkeys (Table 1). The SG $\rightarrow$ IG influence is weak but significant 
$(0.03 ; p<0.01)$ in one penetration and not significant in the other three. Finally, the role of the G layer on the interaction between IG and SG alpha activities was studied by performing conditional causality analysis. After conditioning out the influence of the G layer, the peak $(10 \mathrm{~Hz})$ Granger causality of the IG driving the SG layer is significantly reduced from 0.3 to 0.15 ( $p<$ 0.001) (Fig. 4H). This significant reduction is found in all four penetrations and suggests that part of the IG influence on SG layers could be mediated by the $\mathrm{G}$ layer.

In $\mathrm{V} 2$, the circuit organization underlying the alpha rhythm was found to be nearly identical to that of V4. For the data shown in Figure 2, the power and coherence between the bipolar LFP signals in IG and G layers are shown in Figure 5, $A$ and $B$, respectively. Both power and coherence spectra have peaks in the alpha range with a peak coherence of $0.42(p<0.001)$ at $10 \mathrm{~Hz}$. The bivariate Granger causality spectrum reveals that the IG layers exert a significant causal influence on the $G$ layer with a peak value of $0.46(p<0.001)$ at $9 \mathrm{~Hz}$, and the causal influence in the opposite direction is not significant (Fig. $5 C, D$ ). The IG layers are found to be driving the $G$ layer across all four penetrations in both monkeys (Table 2). In two penetrations, the $\mathrm{G}$ layer has a weak but significant $(0.03, p<0.01 ; 0.05, p<0.01)$ causal influence on the IG layers. The G $\rightarrow$ IG influence is not significant in the other two. Conditioning out the SG layer activity results in a small but significant decrease in IG $\rightarrow$ G causal influence (Fig. $5 D$, Table 2) in two penetrations $(p<0.01)$.

IG and SG layers are coherent $(0.11$ at $10 \mathrm{~Hz} ; p<0.01)$ (Fig. $5 F)$, with IG driving SG with a peak Granger causality value 0.12 $(p<0.001)$ at $10 \mathrm{~Hz}$ (Fig. $5 G, H)$. When activity of the G layer is conditioned out, the strength of the driving from IG to SG decreases significantly $(p<0.001)$ from 0.12 to 0.03 (Fig. $5 H$ ), and the reduction is found to be significant across three penetrations (Table 2). As in V4, the IG layer current is found to be consistently driving the other layers in the alpha frequency range across all four penetrations in both monkeys (Table 2).

In IT, the previous analysis (Fig. 3) revealed a prominent, active alpha current generator in the SG layers and a weak, passive one in the IG layer. Their interaction is studied by subjecting the two bipolar LFP signals to a bivariate MVAR model. The power spectra show a peak at $\sim 11 \mathrm{~Hz}$ (Fig. 6A), and the coherence spectrum has a peak at the same frequency with a peak value of $0.34(p<0.001)$ (Fig. 6B). The Granger causality analysis indicates that the SG layer alpha current exerts a significant causal influence on the IG current at $\sim 10 \mathrm{~Hz}$, where the spectral peak value is $0.29(p<0.001)$ (Fig. $6 C$ ). The causal influence in the opposite direction is not significant (Fig. 6D). In contrast to V4 and V2, the SG layers in IT are found to be consistently driving the IG layers in the alpha frequency range in all four penetrations for both monkeys (Table 3), and the IG $\rightarrow$ SG driving in the alpha range is either not significant or very weak.

Figure 7 gives a summary of the findings presented thus far. The main cell groups in SG, G, and IG layers are schematically represented. The locations of alpha current generators are indicated by green shading, and arrows are used to signify synaptic transmissions as determined by Granger causality. In V4 and V2 (Fig. 7, left and middle), the IG layers contain the primary local alpha pacemaker, and the G layer contains a secondary local pacemaker. The alpha current generator in the SG layers is nonpacemaking. The arrow from G to SG is inferred from conditional Granger causality analysis. In IT (Fig. 7, right), the primary local alpha pacemaker is in the SG layers. The alpha current generator in the IG layers is nonpacemaking. No alpha current is found in the $\mathrm{G}$ layer.

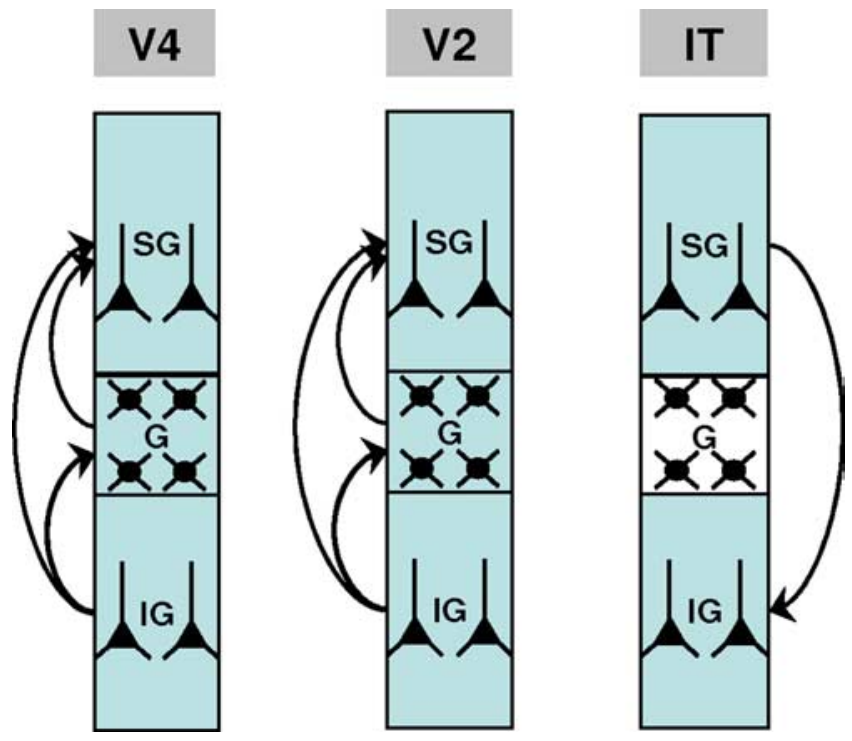

Figure 7. Schematic summarizing the results in V4 (left), V2 (middle), and IT (right). Layers containing alpha current generators are shaded in green. Arrows, depicting the interaction patterns among these generators, are understood in the sense of Granger causality. In V4 and V2, alpha current generators are found in SG, G, and IG layers, with the IG generator acting as the primary local alpha pacemaker. In IT, alpha current generators are found in SG and IG layers, with the SG generator acting as the primary local alpha pacemaker.

The analysis reported here focuses mainly on neural activity in the alpha frequency range $(8-12 \mathrm{~Hz})$. As can be seen in Figures $4-6$, peaks in the beta $(13-30 \mathrm{~Hz})$ and gamma $(>30 \mathrm{~Hz})$ frequency ranges also occur in both coherence and Granger causality spectra. In some instances, neural behavior in these higherfrequency bands contrasts with that in the alpha band (Fig. $5 G, H)$. A separate study is currently underway to elucidate the columnar organization of neural oscillations in the beta and gamma bands, as well as the possible interactions and coupling of activity in these frequency bands (Lakatos et al., 2005; Canolty et al., 2006).

\section{Visual alpha power and auditory task performance}

Prestimulus alpha power and auditory reaction time for the representative V4, V2, and IT penetrations in Figures 1-3 are shown as scatter plots in Figure $8 A-C$, respectively. In Figure $8 A(\mathrm{~V} 4)$, the Spearman rank correlation was $r=-0.69(p<0.01)$. Similarly, in Figure $8 B$ (V2), the Spearman rank correlation was $r=$ $-0.56(p<0.01)$. Such significant negative correlations were found in all four V2 penetrations (Table 4) and three V4 penetrations (Table 4). In the remaining V4 penetration, the correlation was negative but did not reach significance $(r=-0.16 ; p=$ 0.09 ). In contrast to V4 and V2, alpha power in IT showed a positive correlation with auditory RT (Fig. $8 C$ ) in three penetrations. In the remaining penetration, the correlation was also positive but not significant $(r=0.18 ; p=0.1)$. Two additional analyses were considered. First, a contact-by-contact analysis revealed that the alpha power-RT correlation is similar across laminae. No consistent layer specificity was observed. Second, whether a nonlinear relationship may exist between prestimulus alpha power and RT (Linkenkaer-Hansen et al., 2004) was tested by fitting quadratic functions to the scatter plots in Figure 8. In all areas and across all penetrations, such fit was found to be not significant at the $p=0.05$ level. Thus, a linear relationship appears to best characterize the alpha power-RT relationship in our data. 
A

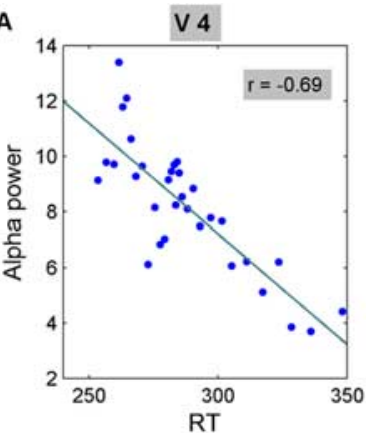

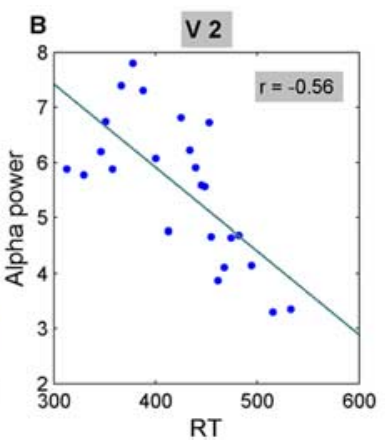

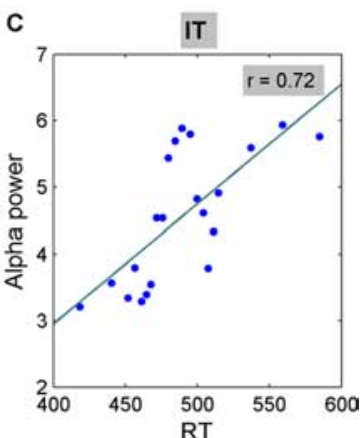

third signal is statistically conditioned out (Geweke, 1984; Chen et al., 2006; Ding et al., 2006). Here, conditioning out the activity of the $\mathrm{G}$ layer revealed a significant decrease of influence from the IG to SG layers. However, the conditional causal influence remained statistically significant, suggesting that the causal influence from the IG to SG layers has both a direct component and a component mediated by the G layer.

The anatomical connectivity within a cortical (Lund, 2002; Douglas and Martin, 2004) column provides the basis for understanding the findings. For excitatory neurons, axons from granular layer cells

Table 4. Spearman rank correlation coefficient values between visual alpha power (averaged across all electrode contacts in a penetration) and auditory reaction time

\begin{tabular}{llll}
\hline Penetration & V2 & V4 & IT \\
\hline 1 & $-0.56^{*}$ & $-0.43^{*}$ & $0.38^{*}$ \\
2 & $-0.45^{*}$ & -0.16 & 0.18 \\
3 & $-0.38^{*}$ & $-0.69^{*}$ & $0.72^{*}$ \\
4 & $-0.12^{*}$ & $-0.29^{*}$ & $0.39^{*}$ \\
\hline${ }^{*} p<0.01$. & & &
\end{tabular}

\section{Discussion}

Neuronal oscillations are ubiquitous in neocortex. Their mechanisms and functions have been investigated most extensively in in vitro slice preparations. Flint and Connors (1996) and Silva et al. (1991) found that layer 5 pyramidal cells in sensory cortices fired rhythmically at rates appropriate for driving the generation of alpha band LFP rhythms, whereas Lukatch and MacIver (1997) concluded that in the entorhinal cortex, alpha rhythms originated in layers $2 / 3$. The present study aimed at resolving this apparent discrepancy, as well as at bridging the gap between in vitro findings and functioning of the intact brain.

Neuronal mechanisms of the alpha rhythm in V2, V4, and IT The spontaneous alpha oscillations in occipital areas V2 and V4 have similar laminar organization. For both areas, current source density analysis revealed alpha currents in IG, G, and SG layers. The depth of the IG current generator corresponds to lamina 5 . The robust CSD-MUA coherence in the IG and G layers suggests that local neuronal activity in these layers could potentially act as pacemakers of alpha rhythms. The alpha current in the SG layers was out of phase with that in IG and G layers, and the CSD-MUA coherence was not significant. A plausible explanation for poor CSD-MUA coherence at the SG layers is dampening caused by inhibition (Schroeder et al., 1995), arising from local inhibitory mechanisms or from inhibitory input to the superficial pyramidal cells from layer 5 interneurons (Dantzker and Callaway, 2000; Bacci et al., 2005; Watts and Thomson, 2005), as specified in a recent model of alpha rhythm (Karameh et al., 2006).

Bivariate Granger causality analysis indicated that in V2 and V4, the IG layer was consistently driving the $\mathrm{G}$ as well as the SG layers, suggesting that the primary local alpha pacemaker was contained in the IG layers. We tested further whether the causal influence from IG to SG layers, revealed by bivariate Granger causality analysis, could be mediated by the $G$ layer. As an alternative to partial coherence analysis (Lopes da Silva et al., 1980; Kocsis et al., 1999), conditional Granger causality tests the directional influences between two signals after the influence from a synapse onto the pyramidal cells in the supragranular layers (2/ 3 ), which in turn send axons that synapse onto infragranular pyramidal neurons. Infragranular pyramidal neurons complete the circuit by sending axons into the granular and upper layers. More recent work has emphasized the role of inhibitory neurons in the genesis of neural oscillations (Buzsáki, 2006). The pertinent projection in this regard is the input of IG layer pyramidal cells to the interneuron populations that inhibit neural activity in SG layers. Consistent with the anatomical pathways, all pairwise coherences, IG-G, IG-SG, and G-SG, are high in the alpha band, indicating synchronized interlaminar oscillations. Our results generalize the in vitro findings (Silva et al., 1991; Flint and Connors, 1996) to the intact brain in awake and behaving monkeys in that in V2 and V4, the alpha rhythm has multiple local cortical generators (Steriade et al., 1990) with a primary local pacemaker at the level of layer 5 .

The laminar organization of alpha rhythm in IT differed from that in V2 and V4. CSD analysis revealed a prominent alpha current generator in the SG layer and a weak one in the IG layer, but none in the G layer. Given that basal SG dendritic arbors are enlarged (up to $400 \mu \mathrm{m}$ ) in IT (Elston et al., 1999), the SG current generator most likely reflects the activity of superficial pyramidal neurons. The laminar LFP power distribution had a maximum over the current generator (Fig. 3B), consistent with this assertion. CSD-MUA coherence was significant in the SG layers. For the IG layer generator, CSD-MUA coherence was not significant. The oscillatory currents in SG and IG layers were highly synchronous, exhibiting significant coherence at $\sim 10 \mathrm{~Hz}$. Granger causality analyses showed that neural activity in the SG layers consistently drives that in the IG layers, indicating that the primary local alpha pacemaker is contained in the SG layers. It is noteworthy that the entorhinal cortex in vitro study by Lukatch and MacIver (1997) suggested that the SG layers might contain the pacemaker of alpha range oscillations. Additionally, in vivo recordings in area IT in macaque monkeys (Shinomoto et al., 2005) found bursting ("clumpy bursty") neurons in layer 3 but not layer 5 .

\section{Cortical heterogeneity and laminar organization of the alpha rhythm}

In the macaque, V2 and V4 are occipital lobe areas, whereas IT is a temporal lobe area. The change in laminar organization of the alpha rhythm as one ascends the ventral pathway calls for a possible explanation. Elston and colleagues (Elston et al., 1999; Elston, 2003) and others (Benavides-Piccione et al., 2002; DeFelipe et al., 2002) have shown that the morphology and spine density of the principal or pyramidal cells differ across cortical areas. Specifically, along the ventral stream of the visual cortex there is a 
progressive increase in cell size, diameter of the dendritic arbor, branching pattern, and spine density of layer 3 pyramidal neurons, leading to their ability to sample inputs from increasingly large numbers of sources. Although layer 5 pyramidal neurons show a similar increase (Elston and Rosa, 2000), the sampling ability of the layer 3 pyramidal cells in higher-order areas (e.g., IT) is further enhanced by a similar increase in the size of the intrinsic "axonal patches" made by neighboring layer 3 pyramidal neurons (Lund et al., 1993; Fujita, 2002). The enhanced sampling ability of superficial pyramidal cells (especially layer 3 ) in IT leads to larger receptive field and increased spontaneous activity relative to deeper layers (Fig. 3B). In lower-order areas, the pyramidal neurons in layer 5 have more extensive horizontal connectivity and stronger integrative properties (Telfeian and Connors, 2003), supporting the observed higher spontaneous activity in deep layers in V4 and V2 (Figs. 1D, 2B). Our results further demonstrate that layers with higher spontaneous activities also contain the pacemakers of the alpha rhythm.

Differences in pyramidal neuron morphology across areas are complemented by differences in distribution and axonal projections of inhibitory neurons (Kritzer et al., 1992; DeFelipe et al., 1999) and in extrinsic connections to the cortical columns (Rockland, 2002). Overall, it seems clear that the intrinsic circuitry of the cortical columns varies across areas (Lund et al., 1981; Elston, 2002), challenging the view that areal functional specificity stems primarily from the differences in patterns of input and output (Mountcastle, 1978; Douglas et al., 1989). Specific aspects of intrinsic circuitry might be important for generating functional specificity within a cortical area. For example, at the top of the hierarchy of the ventral visual stream, the IT area might be critical for the dissemination of top-down attentional and contextual (Gilbert and Sigman, 2007) signals to the lower-order visual and subcortical areas. This could be accomplished by the SG $\rightarrow$ IG driving influence reported here in conjunction with the feedback projections arising from the IG pyramidal neurons.

\section{Alpha rhythm and behavior}

Faster RT for auditory deviant stimulus detection can be considered an index of increased auditory attentiveness, at the expense of visual attention. The negative correlation between alpha power in V4 and V2 and auditory RT (Fig. 8A,B) is consistent with the intermodal attention experiment by Foxe et al. (1998) and the classical reactivity of the alpha rhythm (Shaw, 2003). However, the alpha activity in IT has an entirely different laminar organization from that of V4 and V2, and a positive correlation with auditory RT (Fig. 8C). The fact that the behavioral correlates of alpha rhythms appear different for the lower- and higher-order visual cortices is consistent with the idea that these areas are differentially involved in internally versus externally directed processing states, as defined by Ray and Cole (1985) and Shaw (2003). Critically, the laminar organization of the alpha rhythm in different cortices may be linked to the functions they support.

\section{Implication for interpretation of scalp EEG recordings}

Source localization studies in humans (Hari and Salmelin, 1997; Lopes da Silva, 2004; Feige et al., 2005) have found that the alpha rhythm is maximal along the midline over the posterior-occipital areas. If alpha current generators in human lateral occipital complex (LOC), considered the analog of the macaque IT, have a similar supragranular source/sink/source configuration, the quadrupoles formed by the radially organized dendrites would give rise to a closed field decaying faster than a typical dipole configuration (Mitzdorf, 1985; Lopes da Silva and Rotterdam,
2005). As a result, neural ensembles in LOC and surrounding areas might make a smaller contribution to the scalp alpha rhythm than those in the midline occipital areas. This suggestion has support in recordings from dogs, in which the alpha activity subdurally recorded over the temporal lobe was found to be smaller than that over the occipital lobe (Lopes da Silva et al., 1980). It is also consistent with the finding that phase reset of the alpha oscillation appears to contribute to the P1/N1 complex of the ERP recorded over the posterior midline, but not to that recorded over more lateral scalp regions (Makeig et al., 2004).

\section{References}

Akaike H (1974) A new look at the statistical model identification. IEEE Trans Automat Cont 19:716-723.

Andersen P, Andersson SA (1968) Physiological basis of the alpha rhythm. New York: Appleton-Century-Crofts.

Baccalá LA, Sameshima K (2001) Partial directed coherence: a new concept in neural structure determination. Biol Cybern 84:463-474.

Bacci A, Huguenard JR, Prince DA (2005) Modulation of neocortical interneurons: extrinsic influences and exercises in self-control. Trends Neurosci 28:602-610.

Benavides-Piccione R, Ballesteros-Yáñez I, DeFelipe J, Yuste R (2002) Cortical area and species differences in dendritic spine morphology. J Neurocytol 31:337-346.

Berger H (1929) Über das Elektroencephalogramm des Menschen (On the electroencephalogram of man). Arch Psychiatr Nervenkr 87:527-570.

Bernasconi C, von Stein A, Chiang C, König P (2000) Bi-directional interactions between visual areas in the awake behaving cat. Neuroreport 11:689-692.

Brovelli A, Ding M, Ledberg A, Chen Y, Nakamura R, Bressler SL (2004) Beta oscillations in a large-scale sensorimotor cortical network: directional influences revealed by Granger causality. Proc Natl Acad Sci U S A 101:9849-9854.

Buzsáki G (2006) Rhythms of the brain. New York: Oxford UP.

Canolty RT, Edwards E, Dalal SS, Soltani M, Nagarajan SS, Kirsch HE, Berger MS, Barbaro NM, Knight RT (2006) High gamma power is phaselocked to theta oscillations in human neocortex. Science 313:1626-1628.

Castro-Alamancos MA, Connors BW (1996) Cellular mechanisms of the augmenting response: short-term plasticity in a thalamocortical pathway. J Neurosci 16:7742-7756.

Castro-Alamancos MA, Tawara-Hirata Y (2007) Area-specific resonance of excitatory networks in neocortex: control by outward currents. Epilepsia 48:1572-1584.

Chen CM, Lakatos P, Shah AS, Mehta AD, Givre SJ, Javitt DC, Schroeder CE (2007) Functional anatomy and interactions of fast and slow visual pathways in macaque monkeys. Cereb Cortex 17:1561-1569.

Chen Y, Bressler SL, Ding M (2006) Frequency decomposition of conditional Granger causality and application to multivariate neural field potential data. J Neurosci Methods 150:228-237.

Connors BW, Amitai Y (1997) Making waves in the neocortex. Neuron 18:347-349.

Cooper NR, Croft RJ, Dominey SJJ, Burgess AP, Gruzelier JH (2003) Paradox lost? Exploring the role of alpha oscillations during externally vs. internally directed attention and the implications for idling and inhibition hypotheses. Int J Psychophysiol 47:65-74.

Csicsvari J, Jamieson B, Wise KD, Buzsáki G (2003) Mechanisms of gamma oscillations in the hippocampus of the behaving rat. Neuron 37:311-322.

Dantzker JL, Callaway EM (2000) Laminar sources of synaptic input to cortical inhibitory interneurons and pyramidal neurons. Nat Neurosci 3:701-707.

DeFelipe J, González-Albo MC, Del Río MR, Elston GN (1999) Distribution and patterns of connectivity of interneurons containing calbindin, calretinin, and parvalbumin in visual areas of the occipital and temporal lobes of the macaque. J Comp Neurol 412:515-526.

DeFelipe J, Alonso-Nanclares L, Arellano JI (2002) Microstructure of the neocortex: comparative aspects. J Neurocytol 31:299-316.

Ding M, Bressler SL, Yang W, Liang H (2000) Short-window spectral analysis of cortical event-related potentials by adaptive multivariate autoregressive modeling: data preprocessing, model validation, and variability assessment. Biol Cybern 83:35-45.

Ding M, Chen Y, Bressler SL (2006) Granger causality: basic theory and 
application to neuroscience. In: Handbook of time series analysis (Winterhalder M, Schelter B, Timmer J, eds), pp 437-460. Berlin: Wiley-VCH Verlag.

Douglas RJ, Martin KAC (2004) Neuronal circuits of the neocortex. Annu Rev Neurosci 27:419-451.

Douglas RJ, Martin KAC, Whitteridge D (1989) A canonical microcircuit for neocortex. Neural Comput 1:480-488.

Durbin J, Watson GS (1950) Testing for serial correlation in least squares regression, I. Biometrika 37:409-428.

Efron B (1982) The jackknife, the bootstrap, and other resampling plans. Philadelphia: SIAM.

Elston GN (2002) Cortical heterogeneity: implications for visual processing and polysensory integration. J Neurocytol 31:317-335.

Elston GN (2003) Cortex, cognition and the cell: new insights into pyramidal neuron and prefrontal function. Cereb Cortex 13:1124-1138.

Elston GN, Rosa MG (2000) Pyramidal cells, patches, and cortical columns: a comparative study of infragranular neurons in TEO, TE, and the superior temporal polysensory area of the macaque monkey. J Neurosci 20:RC117(1-5)

Elston GN, Tweedale R, Rosa MGP (1999) Cortical integration in the visual system of the macaque monkey: large-scale morphological differences in the pyramidal neurons in the occipital, parietal and temporal lobes. Proc Biol Sci 266:1367-1374.

Feige B, Scheffler K, Esposito F, Di Salle F, Hennig J, Seifritz E (2005) Cortical and subcortical correlates of electroencephalographic alpha rhythm modulation. J Neurophysiol 93:2864-2872.

Flint AC, Connors BW (1996) Two types of network oscillations in neocortex mediated by distinct glutamate receptor subtypes and neuronal populations. J Neurophysiol 75:951-957.

Foxe JJ, Simpson GV, Ahlfors SP (1998) Parieto-occipital $\sim 10 \mathrm{~Hz}$ activity reflects anticipatory state of visual attention mechanisms. Neuroreport 9:3929-3933.

Freiwald WA, Valdes P, Bosch J, Biscay R, Jimenez JC, Rodriguez LM, Rodriguez V, Kreiter AK, Singer W (1999) Testing non-linearity and directedness of interactions between neural groups in the macaque inferotemporal cortex. J Neurosci Methods 94:105-119.

Fujita I (2002) The inferior temporal cortex: architecture, computation, and representation. J Neurocytol 31:359-371.

Geweke J (1982) Measurement of linear-dependence and feedback between multiple time-series. J Am Stat Assoc 77:304-313.

Geweke J (1984) Measures of conditional linear-dependence and feedback between time-series. J Am Stat Assoc 79:907-915.

Gilbert CD, Sigman M (2007) Brain states: top-down influences in sensory processing. Neuron 54:677-696.

Givre SJ, Schroeder CE, Arezzo JC (1994) Contribution of extrastriate area V4 to the surface-recorded flash VEP in the awake macaque. Vision Res 34:415-428.

Granger CWJ (1969) Investigating causal relations by econometric models and cross-spectral methods. Econometrics 37:424-438.

Hari R, Salmelin R (1997) Human cortical oscillations: a neuromagnetic view through the skull. Trends Neurosci 20:44-49.

Hendry SHC, Fuchs J, deBlas AL, Jones EG (1990) Distribution and plasticity of immunocytochemically localized GABAA receptors in adult monkey visual cortex. J Neurosci 10:2438-2450.

Hesse W, Möller E, Arnold M, Schack B (2003) The use of time-variant EEG Granger causality for inspecting directed interdependencies of neural assemblies. J Neurosci Methods 124:27-44.

Jones EG (1990) Determinants of the cytoarchitecture of the cerebral cortex. In: Signal and sense: local and global order in perceptual maps (Edelman GM, Gall WE, Cowan WM, eds), pp 3-49. New York: Wiley.

Jones SR, Pinto DJ, Kaper TJ, Kopell N (2000) Alpha-frequency rhythms desynchronize over long cortical distances: a modeling study. J Comput Neurosci 9:271-291.

Karameh FN, Dahleh MA, Brown EN, Massaquoi SG (2006) Modeling the contribution of lamina 5 neuronal and network dynamics to low frequency EEG phenomena. Biol Cybern 95:289-310.

Kocsis B, Bragin A, Buzsáki G (1999) Interdependence of multiple theta generators in the hippocampus: a partial coherence analysis. J Neurosci 19:6200-6212.

Kritzer MF, Cowey A, Somogyi P (1992) Patterns of inter- and intralaminar GABAergic connections distinguish striate (V1) and extrastriate (V2, V4) visual cortices and their functionally specialized subdivisions in the rhesus monkey. J Neurosci 12:4545-4564.

Lakatos P, Shah AS, Knuth KH, Ulbert I, Karmos G, Schroeder CE (2005) An oscillatory hierarchy controlling neuronal excitability and stimulus processing in the auditory cortex. J Neurophysiol 94:1904-1911.

Lakatos P, Chen CM, O'Connell MN, Mills A, Schroeder CE (2007) Neuronal oscillations and multisensory interaction in primary auditory cortex. Neuron 53:279-292.

Lakatos P, Karmos G, Mehta AD, Ulbert I, Schroeder CE (2008) Oscillatory entrainment as a mechanism of attentional selection. Science 320:110-113.

Lehtelä L, Salmelin R, Hari R (1997) Evidence for reactive magnetic 10-Hz rhythm in the human auditory cortex. Neurosci Lett 222:111-114.

Le Van Quyen M, Bragin A (2007) Analysis of dynamic brain oscillations: methodological advances. Trends Neurosci 30:365-373.

Liang H, Ding M, Nakamura R, Bressler SL (2000) Causal influences in primate cerebral cortex during visual pattern discrimination. Neuroreport 11:2875-2880

Linkenkaer-Hansen K, Nikulin VV, Palva S, Ilmoniemi RJ, Palva JM (2004) Prestimulus oscillations enhance psychophysical performance in humans. J Neurosci 24:10186-10190.

Lipton ML, Fu K-MG, Branch CA, Schroeder CE (2006) Ipsilateral hand input to area $3 \mathrm{~b}$ revealed by converging hemodynamic and electrophysiological analyses in macaque monkeys. J Neurosci 26:180-185.

Lopes da Silva FH (1991) Neural mechanisms underlying brain waves: from neural mechanisms to networks. Electroencephalogr Clin Neurophysiol 79:81-93.

Lopes da Silva FH (2004) Functional localization of brain sources using EEG and/or MEG data: volume conductor and source models. Magn Reson Imaging 22:1533-1538.

Lopes da Silva FH, Rotterdam AV (2005) Biophysical aspects of EEG and magnetoencephalogram generation. In: Electroencephalography: basic principles, clinical applications and related fields (Niedermeyer E, ed), pp 93-109. Baltimore: Williams and Wilkins.

Lopes da Silva FH, van Leeuwen WS (1977) The cortical source of the alpha rhythm. Neurosci Lett 6:237-241.

Lopes da Silva FH, van Lierop TH, Schrijer CF, van Leeuwen WS (1973a) Organization of thalamic and cortical alpha rhythms: spectra and coherences. Electroencephalogr Clin Neurophysiol 35:627-639.

Lopes da Silva FH, van Lierop TH, Schrijer CF, van Leeuwen WS (1973b) Essential differences between alpha rhythms and barbiturate spindles: spectra and thalamo-cortical coherences. Electroencephalogr Clin Neurophysiol 35:641-645.

Lopes da Silva FH, Vos JE, Mooibroek J, Van Rotterdam A (1980) Relative contributions of intracortical and thalamo-cortical processes in the generation of alpha rhythms, revealed by partial coherence analysis. Electroencephalogr Clin Neurophysiol 50:449-456.

Lukatch HS, MacIver MB (1997) Physiology, pharmacology, and topography of cholinergic neocortical oscillations in vitro. J Neurophysiol 77:2427-2445.

Lund JS (2002) Specificity and non-specificity of synaptic connections within mammalian visual cortex. J Neurocytol 31:203-209.

Lund JS, Hendrickson AE, Ogren MP, Tobin EA (1981) Anatomical organization of primate visual area VII. J Comp Neurol 202:19-45.

Lund JS, Yoshioka T, Levitt JB (1993) Comparison of intrinsic connectivity in different areas of macaque monkey cerebral cortex. Cereb Cortex 3:148-162.

Lutkepohl H (2005) A new introduction to multiple time series analysis. New York: Springer.

Makeig S, Debener S, Onton J, Delorme A (2004) Mining event-related brain dynamics. Trends Cogn Sci 8:204-210.

Mehta AD, Ulbert I, Schroeder CE (2000a) Intermodal selective attention in monkeys. I: Distribution and timing of effects across visual areas. Cereb Cortex 10:343-358.

Mehta AD, Ulbert I, Schroeder CE (2000b) Intermodal selective attention in monkeys. II: Physiological mechanisms of modulation. Cereb Cortex 10:359-370.

Mitzdorf U (1985) Current source density method and application in cat cerebral cortex: investigation of evoked potentials and EEG phenomena. Physiol Rev 65:37-100.

Mountcastle VB (1978) An organizing principle for general cortical func- 
tion: The unit module and the distributed system. In: The mindful brain (Schmitt FO, ed), pp 7-50. Cambridge: MIT.

Nalatore H, Ding M, Rangarajan G (2007) Mitigating the effects of measurement noise on Granger causality. Phys Rev E Stat Nonlin Soft Matter Phys 75:031123.

Nicholson C (1973) Theoretical analysis of field potentials in anisotropic ensembles of neuronal elements. IEEE Trans Biomed Eng 20:278-288.

Niedermeyer E (2005) The normal EEG of the waking adult. In: Electroencephalography: basic principles, clinical applications and related fields (Niedermeyer E, ed), pp 149-173. Baltimore: Williams and Wilkins.

Okatan M, Wilson MA, Brown EN (2005) Analyzing functional connectivity using a network likelihood model of ensemble neural spiking activity. Neural Comput 17:1927-1961.

Palva S, Palva JM (2007) New vistas for $\alpha$-frequency band oscillations. Trends Neurosci 30:150-158.

Ray WJ, Cole HW (1985) EEG alpha activity reflects attentional demands and beta activity reflects emotional and cognitive processes. Science 228:750-752.

Rockland KS (2002) Non-uniformity of extrinsic connections and columnar organization. J Neurocytol 31:247-253.

Schroeder CE, Tenke CE, Givre SJ, Arezzo JC, Vaughan HG Jr (1991) Striate cortical contribution to the surface-recorded pattern-reversal VEP in the alert monkey. Vision Res 31:1143-1157.

Schroeder CE, Tenke CE, Givre SJ (1992) Subcortical contributions to the surface-recorded flash-VEP in the awake macaque. Electroencephalogr Clin Neurophysiol 84:219-231.

Schroeder CE, Steinschneider M, Javitt DC, Tenke CE, Givre SJ, Mehta AD, Simpson GV, Arezzo JC, Vaughan HG Jr (1995) Localization of ERP generators and identification of underlying neural processes. Electroencephalogr Clin Neurophysiol Suppl 44:55-75.

Schroeder CE, Mehta AD, Givre SJ (1998) A spatiotemporal profile of visual system activation revealed by current source density analysis in the awake macaque. Cereb Cortex 8:575-592.
Shah AS, Bressler SL, Knuth KH, Ding M, Mehta AD, Ulbert I, Schroeder CE (2004) Neural dynamics and the fundamental mechanisms of eventrelated brain potentials. Cereb Cortex 14:476-483.

Shaw JC (2003) Brain's alpha rhythm and the mind. Amsterdam: Elsevier.

Shinomoto S, Miyazaki Y, Tamura H, Fujita I (2005) Regional and laminar differences in in vivo firing patterns of primate cortical neurons. J Neurophysiol 94:567-575.

Silva LR, Amitai Y, Connors BW (1991) Intrinsic oscillations of neocortex generated by layer 5 pyramidal neurons. Science 251:432-435.

Steriade M (2004) Neuronal cell classes are flexible entities. Nat Rev 5:121-134.

Steriade M, Gloor P, Llinás RR, Lopes da Silva FH, Mesulam MM (1990) Basic mechanisms of cerebral rhythmic activities. Electroencephalogr Clin Neurophysiol 76:481-508.

Telfeian AE, Connors BW (2003) Widely integrative properties of layer 5 pyramidal cells support a role for processing extralaminar synaptic inputs in rat neocortex. Neurosci Lett 343:121-124.

Tenke CE, Schroeder CE (1990) A model for sublaminar and thalamocortical contributions to the surface VEP: implications for current source density (CSD) analysis. Soc Neurosci Abstr 16:1.569.

Truccolo W, Eden UT, Fellows MR, Donoghue JP, Brown EN (2005) A point process framework for relating neural spiking activity to spiking history, neural ensemble, and extrinsic covariate effects. J Neurophysiol 93:1074-1089.

Watts J, Thomson AM (2005) Excitatory and inhibitory connections show selectivity in the neocortex. J Physiol 562:89-97.

Wiener N (1956) The theory of prediction. In: Modern mathematics for the engineer (Beckenbach EF, ed), Chap 8. New York: McGraw-Hill.

Worden MS, Foxe JJ, Wang N, Simpson GV (2000) Anticipatory biasing of visuospatial attention indexed by retinotopically specific alpha-band electroencephalography increases over occipital cortex. J Neurosci 20: RC63(1-6). 